\title{
Chapter 1 \\ Experimental and Simulated Electron \\ Microscopy in the Study of Metal \\ Nanostructures
}

\author{
Sergio Mejía-Rosales and Miguel José-Yacamán
}

\subsection{Introduction}

One of the most exciting features of nanostructures relays on the fact that matter organized as objects at the nanoscale may have chemical and physical properties different from those of the same material in its common bulk presentation. This sizedependent properties are related to several physical phenomena, such as quantum behavior, high surface-to-volume ratio, and thermal effects [1,2]. Far from being an unfortunate fact, the unique properties of nanostructures may in principle be fine-tuned to be used for a specific purpose, but to be able to dominate matter at this range of sizes, high precision tools must be used in order to investigate, measure, and modify the physical and chemical characteristics of the nanostructures. The problem is similar to the one of constructing a phase diagram for the bulk materials, but taking also into consideration size and shape, besides temperature and crystalline structure. Several groups have concentrated some of their efforts to this task (with varying degrees of success), but these attempts are inherently incomplete or, at best, restricted to one specific material and to a narrow range of sizes and temperatures $[3,4]$. On the other hand, these studies are generally based either on theoretical results or on indirect experimental measurements, and there is just a relatively small number of papers that concentrate on first-hand measurements of the detailed crystal structure and local chemical composition of the nanostructures. This is, to certain degree, understandable: A detailed analysis of the structure and composition

\footnotetext{
S. Mejía-Rosales ( $\square)$

Center for Innovation and Research in Engineering and Technology, and CICFIM-Facultad de Ciencias Físico-Matemáticas, Universidad Autónoma de Nuevo León, San Nicolás de los Garza, NL 66450, México

e-mail: sergio.mejiars@uanl.edu.mx

M. José-Yacamán

Department of Physics and Astronomy, University of Texas at San Antonio, One UTSA Circle, San Antonio, TX 78249, USA

e-mail: miguel.yacaman@utsa.edu
} 


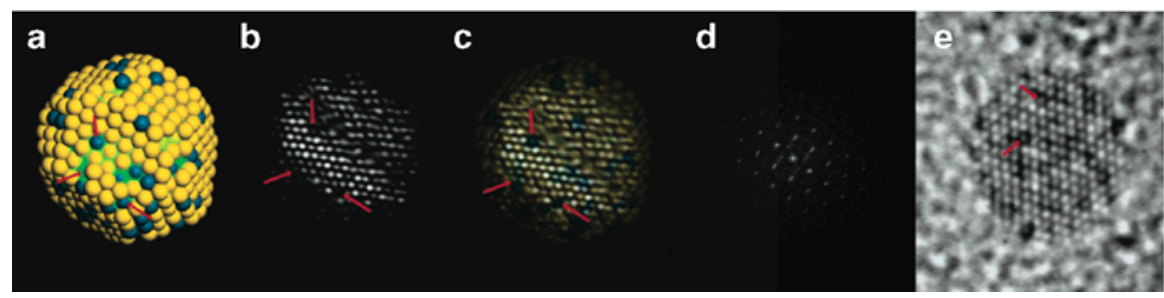

Fig. 1.1 (a) Model of AuPd particle of 923 atoms used to simulate the features of the particle shown in (e); (b) the corresponding simulated TEM image; (c) overlap of parts (a) and (b); (d) calculated FFT pattern for the simulated particle. In the model, blue spheres represent Pd atoms, and yellow spheres correspond to Au atoms. Reprinted with permission from [6]. Copyright 2007 American Chemical Society

requires sub-nanometric resolution, and the electron microscopy facilities capable of reaching this resolution are not always easy to access. Besides, the interpretation of the electron micrographs is not always straightforward, since the intensity signal that correlates with the atomic positions depends not only on the length of the atomic columns parallel to the direction of the electron beam, but also on the chemical species, and on the microscope's parameters at which the micrograph was obtained [5]. Thus, the solution to the problem of extracting a third dimension from the information contained in a strictly two-dimensional image has to be similar to the one that the human mind uses to recognize macroscopic objects sensed by the naked eye: through (a) the comparison of the observed patterns against simple models acquired by previous experience or inherited, and (b) the rough estimation of the probability of a particular model to correspond to the pattern, taking into consideration a small set of rules. An example of this use is shown in Fig. 1.1, taken from [6]. Here, a TEM micrograph of a gold-palladium nanoparticle of $2 \mathrm{~nm}$ of diameter shows that some specific spots on the particle have a different intensity from the rest (Fig. 1.1e); a model is proposed in Fig. 1.1a, and a simulated TEM micrograph, shown in Fig. 1.1b, is obtained. The image of the ball-and-stick model is superposed on the simulated TEM image (Fig. 1.1c), and a correlation is found between the position of the spots and some specific sites at the surface of the particle, where a Pd atom is surrounded by Au atoms that are not in the same plane of the $\mathrm{Pd}$ atom. It is likely that these specific isolated Pd sites have a relevant effect on the chemical activity of the particle, and at least one theoretical study has investigated these kind of sites [7].

In Fig. 1.1, as in the other figures where we present simulated TEM micrographs, we used the SimulaTEM program developed by Gómez-Rodríguez et al. that, unlike other programs with similar purposes, allows non-periodic structures as input [8]. The principles underlying the creation of this kind of simulated TEM micrographs (and also the real ones) will be discussed in the following sections.

This chapter deals with the specific issues that need to be considered in order to make an adequate interpretation of the micrograph of a nanostructure, with a particular emphasis in the imaging of nanoalloys. We will concentrate in the use of high angular annular dark field scanning transmission electron microscopy 
(HAADF-STEM) imaging, since in this technique the electron signal is strongly dependent on $Z$, the atomic number of the atoms that form the structure [9], which makes it the most appropriate choice for the study of metal nanoparticles.

This review does not intend to be a complete essay on the simulation of electron microscopy but a hands-on guide for researchers interested in the generation of high resolution electron micrographs of metal nanostructures. In accordance with this approach, we will only discuss briefly the working principles of electron microscopy, the role of aberration correctors, and how the theory supporting the imaging of micrographs can be used to simulate the imaging process, to concentrate later on the practical issues to be taken into consideration in the comparison and interpretation of real and simulated electron micrographs of nanostructures. The first sections give a comprehensive (but incomplete) review of the fundamental concepts related to the TEM and HAADF-STEM techniques, and to the multislice method of simulation. The remaining sections will discuss the use of real and simulated HAADF-STEM micrographs for the recognition of nanoalloys, using for this purpose several examples of shapes, sizes, and compositions.

\subsection{The Transmission Electron Microscope in a Nutshell}

In order to study the structure of matter at Angstrom and even sub-Angstrom resolution, a beam with a wavelength on the same order of magnitude is needed. The electron microscope accelerates electrons with a potential difference of several hundreds of $\mathrm{kV}$ in order to produce a beam with this range of energies. In modern electron microscopes, the electrons are usually produced by a cold field-emission gun. The beam is filtered to make it practically monochromatic, and a system of condenser lenses narrows the beam divergence angle. The resulting beam interacts with the specimen, and the transmitted beam is collected by an objective lens and projected to the image plane, normally a ccd array. A simplified schematic diagram is shown in Fig. 1.2.

The electron beam interaction with the sample is better understood if one considers the sample electrostatic potential or, more specifically, the projected potential of the sample. Let's assume as a starting point that the sample is a periodic crystal, and that its electrostatic potential is described by $\phi(r)$. The projected potential will of course depend on the structural information of the sample, and also on the temperature. Then, the interaction of an electron of relativistic mass $m$ with the sample is described by

$$
\left[-\frac{h^{2}}{8 \pi^{2} m} \nabla^{2}-e \phi(r)\right] \Psi(r)=E \Psi(r),
$$

with the original electron wave described by a simple plane wave:

$$
\Psi_{0}(r)=\exp \left\{\mathrm{i}\left(\omega t-2 \pi k_{0} \cdot r\right)\right\} .
$$


Fig. 1.2 An overly simplified diagram of the conventional transmission electron microscope

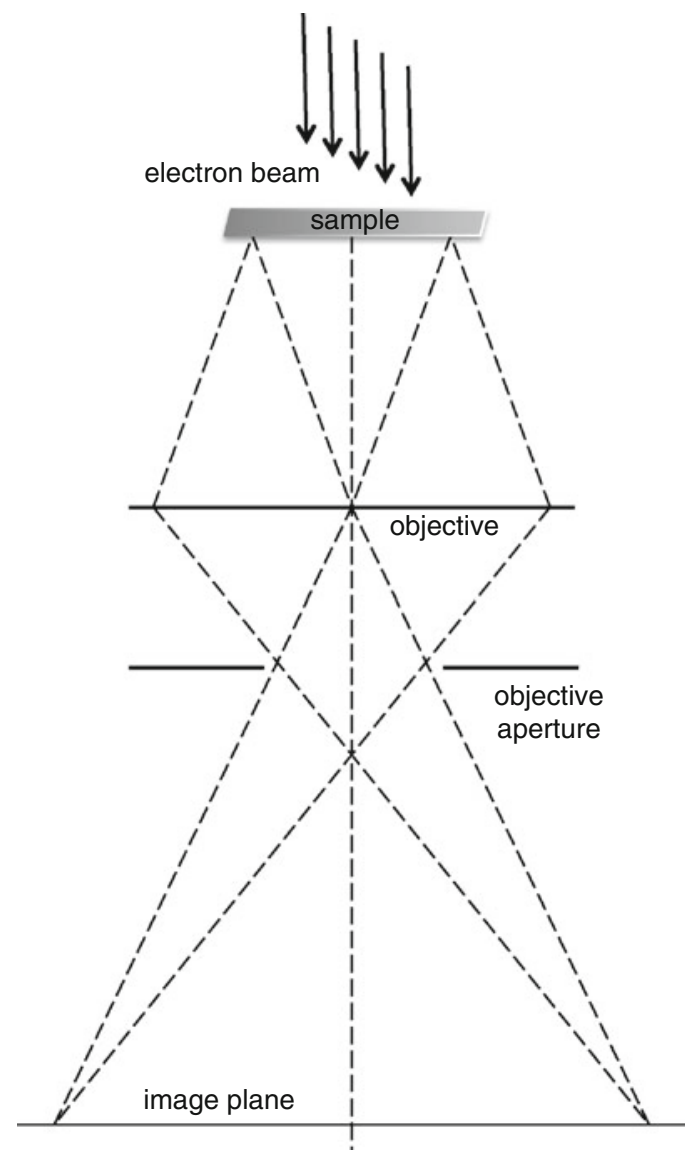

The solution to the Schrodinger equation will describe the electron wave function after interacting with the sample. Even with the periodicity assumption, the solution cannot be found directly without making additional simplifications. We will come back to this point in the section devoted to approximation methods of calculation.

The objective lens, located after the sample in a TEM, focuses the electron beam, which mathematically can be described by the Fourier transform of the wave at the exit of the sample. The objective lens is far from a perfect lens, and it will introduce aberrations in the image-plane wavefunction (the optical elements after the condenser lens, in charge of the amplification, won't produce important aberrations to the image). High resolution microscopes include in their design aberration correctors that make sub-Angstrom resolution possible. In an ideal microscope, without any aberrations, the image observed will be directly related to the squared modulus of the image-plane wavefunction [5]. 


\subsection{Aberrations}

Aberrations will occur, though, and this implies that not all the Fourier components of the wave function will transfer equally. The contrast transfer function (CTF) is a broadly used quantity that determines the range of characteristic spatial frequencies that can be directly interpreted in a TEM image. In other words, the CTF imposes the conditions at which "what you see is what you get." In a concise way, the electron wave function at the image plane will be related to the CTF applied to the wavefunction just at the exit of the sample. Objective apertures will affect the $\mathrm{CTF}$, as well as the spatial coherence of the electron beam and its wavelength:

$$
\operatorname{CTF}(k)=-\sin \left[\frac{\pi}{2} C_{\mathrm{s}} \lambda^{3} k^{4}+\pi \Delta z \lambda k^{2}\right] .
$$

Here, $C_{\mathrm{s}}$ is the spherical aberration of the objective lens, and $\Delta z$ is its defocus. The special case where

$$
\Delta z=-\sqrt{4 / 3 C_{\mathrm{s}} \lambda}
$$

is known as the Scherzer condition, and at Scherzer defocus the CTF does not change its sign in a large spatial frequency range, and hence all distances in this range can be interpreted directly as they appear in the image $[5,10]$. Figure 1.3 shows the plot of two CTF, calculated for two different values of $C_{\mathrm{s}}$. Both graphs were calculated at the same voltage $(200 \mathrm{kV})$. The dark blue CTF was generated

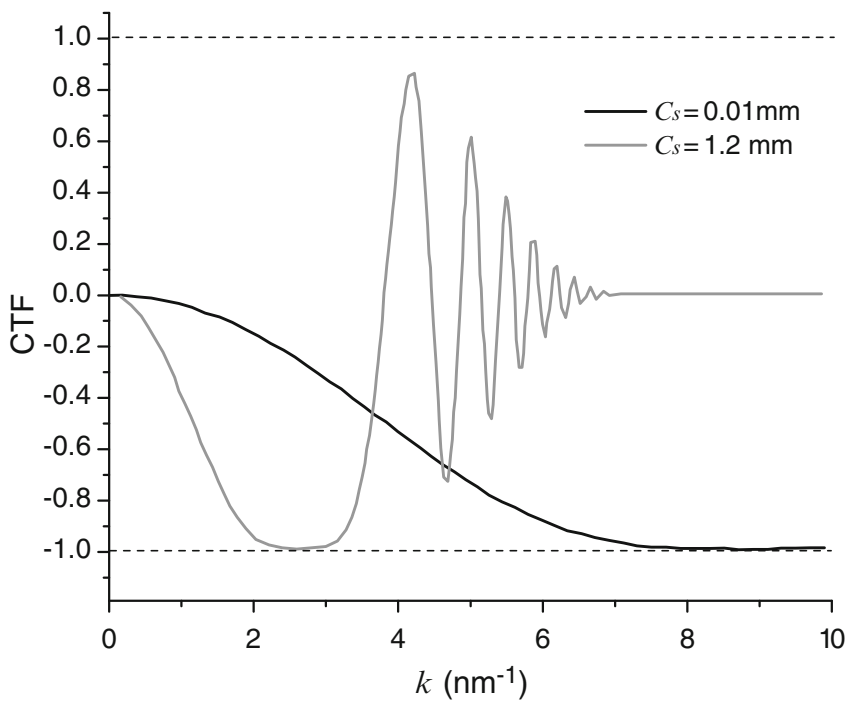

Fig. 1.3 Contrast transfer functions (CTF) for two different values of $C_{\mathrm{s}}$. The darker line represents a CTF calculated at a $C_{\mathrm{s}}$ of $0.01 \mathrm{~mm}$, and the lighter wavy line was calculated at a $C_{\mathrm{s}}$ of $1.2 \mathrm{~mm}$. Both CTFs were obtained at Scherzer conditions 

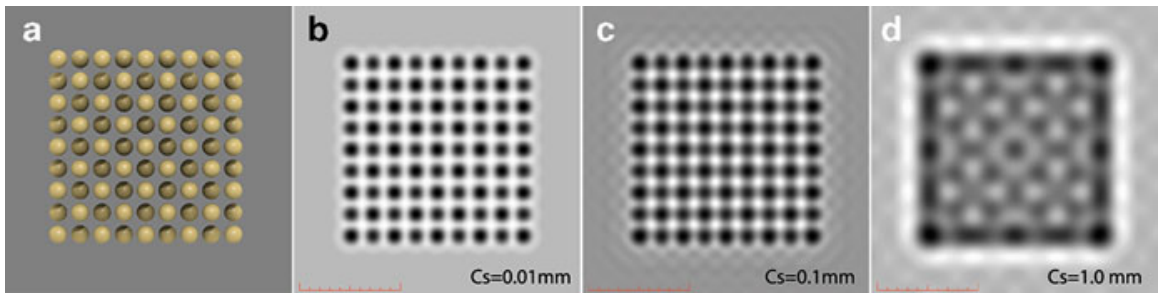

Fig. 1.4 HRTEM simulation of a cubic fcc gold nanostructure obtained at three different values of $C_{\mathrm{s}}$ aberration; (a) Toy representation of the nanostructure, and simulated TEM micrographs with (b) $0.01 \mathrm{~mm}$ of $C_{\mathrm{s}}$ aberration; (c) $0.1 \mathrm{~mm}$ of $C_{\mathrm{s}}$ aberration; (c) $1.0 \mathrm{~mm}$ of $C_{\mathrm{s}}$ aberration. All the micrographs were simulated at the Scherzer condition

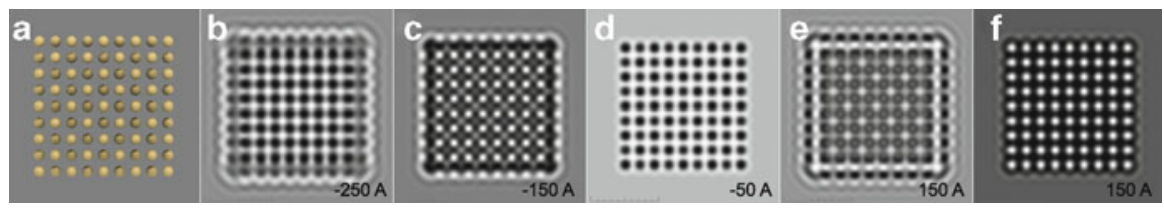

Fig. 1.5 HRTEM simulations of a cubic fcc gold nanostructure calculated at different defocus values. The image labeled as (d) corresponds to the Scherzer defocus

for a $C_{\mathrm{s}}$ of just $0.01 \mathrm{~mm}$, trying to simulate the conditions in a $C_{\mathrm{s}}$-corrected TEM. In this virtual TEM, the theoretical resolution is of $0.08 \mathrm{~nm}$. The light blue CTF was calculated with a $C_{\mathrm{S}}$ of $1.2 \mathrm{~mm}$, for comparison purposes. In this case, the theoretical resolution is $0.25 \mathrm{~nm}$, not quite good for the measurement of interatomic distances. Both CTFs were obtained at Scherzer conditions. The curves were plotted using the CtfExporer software created by M. V. Sidorov [11].

The generation of TEM images at the Scherzer condition allows that atomic positions to correspond with dark spots in the micrograph, if the beam is parallel to the axis zone and the aberrations are small. The effect of aberrations in the resolution should be obvious from the CTF, but it is always interesting to see this effect directly on the images, real or simulated. A comparison of three simulated TEM micrographs is made in Fig. 1.4 for the same sample, a small fcc cubic volume of gold atoms. The figure shows that even at Scherzer defocus the images are difficult to interpret if aberrations are not as small as in Fig. 1.4b, and that the effect of spherical aberration may appear particularly remarked at the border of the structure.

Figure 1.5 shows the results of conventional HRTEM simulations of a section of a gold lattice at different values of defocus, Scherzer included (-50 Angstrom). The voltage was $200 \mathrm{kV}$ at all cases, and a small $C_{\mathrm{s}}$ aberration of $0.01 \mathrm{~mm}$ was used, with zero astigmatism. At Scherzer defocus, the atomic columns are completely resolved, even at the surface of the structure. 


\subsection{High Angle Annular Dark Field Scanning Transmission Electron Microscope}

From the point of view of the reciprocity theorem, the STEM is optically equivalent to an inverted TEM, in the sense that if the source and the detector exchange positions, the electron ray paths remain the same [10]. In the STEM, the objective lens - and all the relevant optics - is positioned before the specimen (see Fig. 1.6). For the specific case of the Annular Dark Field (ADF) detector, only the electrons scattered between $\theta_{1} \leq \theta \leq \theta_{2}$ contribute to the image, since these are the electrons that reach the annular detector. Working at $200 \mathrm{kV}$ (appropriate for imaging metals), in a typical HAADF-STEM microscope the inner angle $\theta_{1}$ of the ADF detector is set around $50 \mathrm{mrad}$, and the outer angle $\theta_{2}$ is set around 100-200 mrad. At these range of voltages, the electrons momentum is large enough to require a relativistic treatment, and the use of Schröedinger equation to analyze the system is not completely correct without some adjustments at the values of mass and wavelength (strictly speaking, one should use the relativistic Dirac equation instead of Schröedinger's, but this would greatly complicate the already nontrivial set of equations.)

Fig. 1.6 Simplified diagram representing the functioning principle of the

HAADF-STEM microscope

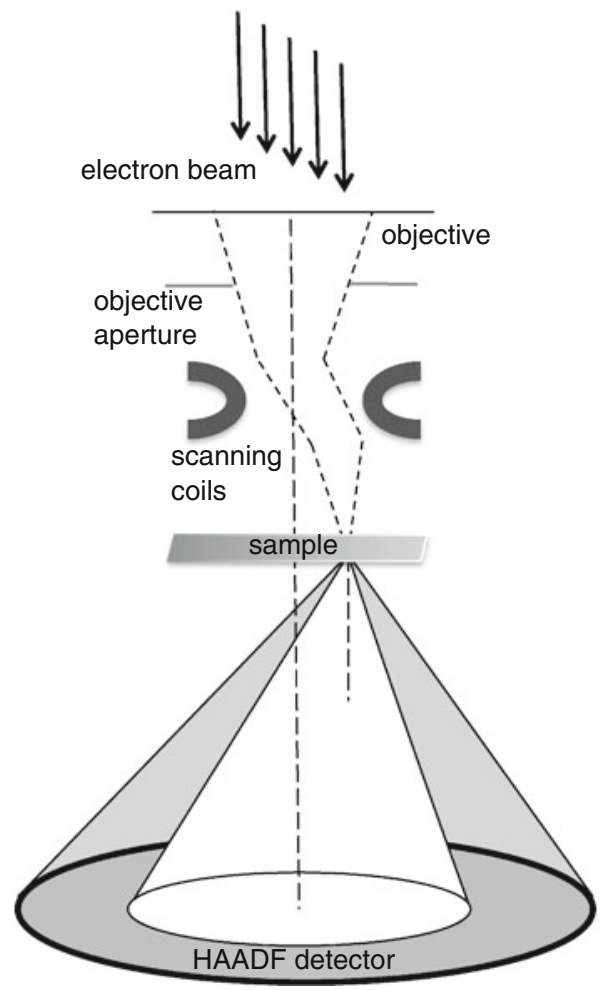


STEM differs from conventional TEM in that, unlike conventional TEM, the electron beam interacts only with a small section of the sample at a time, and the scan process is the one in charge of generating the image as a whole.

But even before taking care of the scanning issue, it is appropriate to review the different methods used to simulate the imaging process of a single area section of the sample. Some approximations are needed in each of these methods, and here we will present them in a succinct way. The reader is encouraged to review elsewhere the details of the methods and the justifications behind the approximations [10].

\subsection{Approximation Methods}

In the Weak Phase Object (WPO) approximation, useful for very thin samples, it is assumed that the electrons are scattered only once by the sample, so the projected potential can be averaged. When the observed sample can be regarded as a WPO, the image is linearly related to the object wavefunction. Even though this is not strictly the case in nanometer-sized structures, we will nevertheless follow this approach, and additional considerations will be taken later.

First, we can assume that the linear image approximation is valid, which means that we take as valid that the intensity $g(r)$ on the image is obtained by a linear convolution of the ideal image of the sample $f(r)$ with the point spread function of the microscope $h(r)$ :

$$
g(r)=\int f\left(r^{\prime}\right) h\left(r-r^{\prime}\right) \mathrm{d} r^{\prime}=f(r) \otimes h\left(r-r^{\prime}\right)
$$

or, in reciprocal space,

$$
G(u)=H(u) F(u) .
$$

$F(u)$ is the Fourier transform of the specimen function, and $H(u)$ is the CTF. The reciprocal vector $\mathbf{u}$ is the spatial frequency. The fact that the intensity on the image is the convolution of $f(r)$ and $h(r)$ means that each point in the image is a collective result of the whole of the sample.

In the WPO approximation, the specimen function is approximated by

$$
f(x, y)=1-\mathrm{i} \sigma V_{t}(x, y)
$$

where the projected potential $V_{t}(x, y)$ is very small since the sample is very thin. But of course, this is not the case in the imaging of metal nanoparticles.

In the Bloch Wave Approximation, the electron wavefunctions are considered as linear combination of Bloch waves, and each reflection is considered explicitly. The BWA is extensively used in periodic systems, but the computing time scales at $N^{3}$, where $N$ is the number of Bloch waves. Several simulation packages, such as JEMS, have implemented BWA in an efficient manner [12]. 


\subsection{The Multislice Method}

The basic idea underlying the multislice method is that the potential of the sample can be approximated by defining a number of slices of thickness $\mathrm{d} z$, and projecting the potential due to the atoms of a particular slice to the central plane of this slice (see Fig. 1.7).

Thus, a solution to the electron wavefunction is obtained for one slice, and used as input for the calculation of the next slice [13]. This means that the choice of very thick slices will speed up the calculations, but the potential function will be poorly approximated. On the other hand, the use of very thin slices will improve the calculation of the projected potential, but the errors due to approximations will accumulate and undermine the final result. The appropriate choice of the number of slices is an issue that requires a generous amount of effort and computing time, and the final decision must consider the benefits of a fast calculation (STEM simulations by the very nature of the technique are computationally expensive and the computing time depends on both the number of slices and the scan resolution) against the benefits of a very precise calculation (that may be critical in quantitative

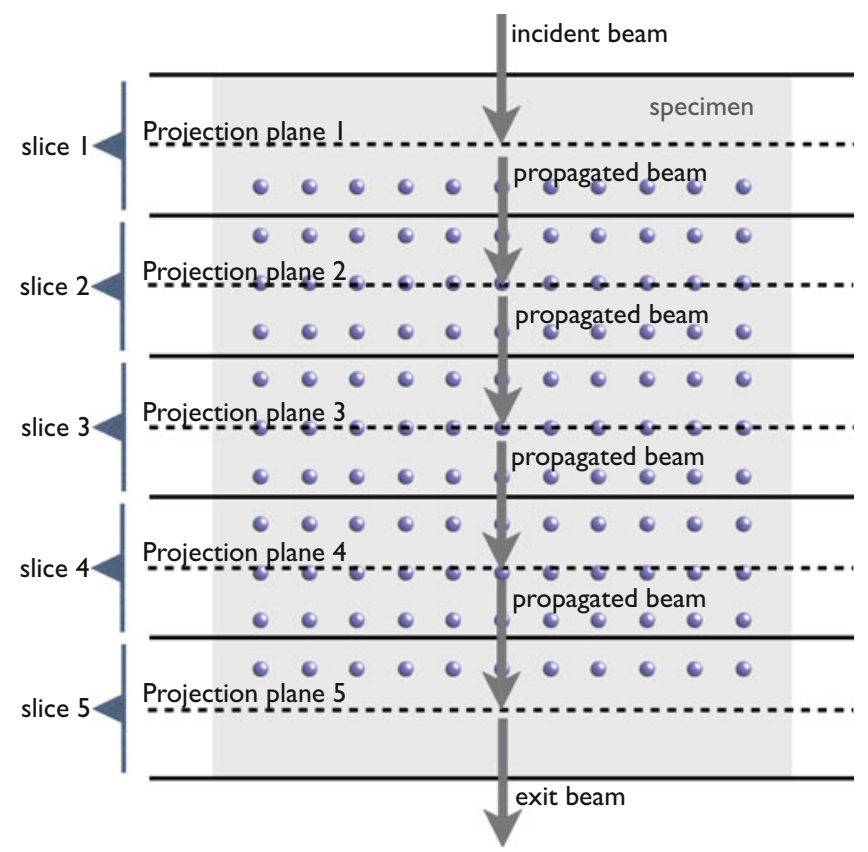

Fig. 1.7 The principle of the multislice method. The sample is sliced, and the potential due to the atoms of a particular slice is projected on a plane; the electron wavefunction corresponding to that slice is then used as input for the interaction with the next slice 
STEM). As pointed out by Ishizuka [14], the multislice method requires several approximations that could be critical in the interpretation of results for inclined illumination, so the orientation of the sample may also affect the final result.

\subsection{STEM Intensity Dependence on Atomic Number}

In a series of papers in the 1970s by Crewe et al., it was found that the annular detector in a HAADF-STEM microscope collects a large amount of the elastic scattered electrons, in such a way that the intensity of the signal collected by the HAADF detector will have a dependence on the scattering cross section, and thus on the atomic number of the atoms present in the sample [15]. Pennycook, one of the pioneers in the technique, gives arguments to this dependence to be close to $\mathrm{Z}^{3 / 2}$ (see [9]). In order to investigate how the intensity signal generated by the HAADF detector depends on the atomic number of a column formed by just one atom of a specific element, we performed a simulation of HAADF for a system of 16 isolated atoms laying on a plane perpendicular to the microscope column. The resulting image is shown in Fig. 1.8, where the positions of the atoms are labeled by the symbols of their respective chemical element.

The HAADF image intensity measured over a line that passes through the center of the atomic positions is shown in Fig. 1.9. Here, it can easily be noted that the contrast between heavy and light atoms is remarkably high.

The maxima in Fig. 1.9 are plotted against the atomic numbers in Fig. 1.10, and a least squares adjustment shows that the HAADF signal follows approximately a $Z^{1.46}$ relation. This dependence is very close to the prediction of Pennycook.

All of the STEM simulations shown and discussed in this chapter, including the simulations presented in the next sections, were made using the commercial version of the xHREM package, a proprietary software designed and coded by Ishizuka [14]. The xHREM suite uses an algorithm based on the Fast Fourier Transform method, and it allows the use of a large number of slices, as many as 1,000 in the latest editions.

\subsection{The Interpretation of an Image}

How can we infer the shapes, sizes, and composition of the objects studied under the electron microscope? The problem of interpretation of electron micrographs is similar to the problem that the human mind has to solve to make a correct interpretation of the two-dimensional images imprinted on the retina. As the experimental psychologist Steven Pinker puts it: Optics is easy but inverse optics impossible [16]. The solution to this factual impossibility lays on the capacity of the human mind to make educated assumptions about the observed objects and their surroundings, or, as Pinker explains, 


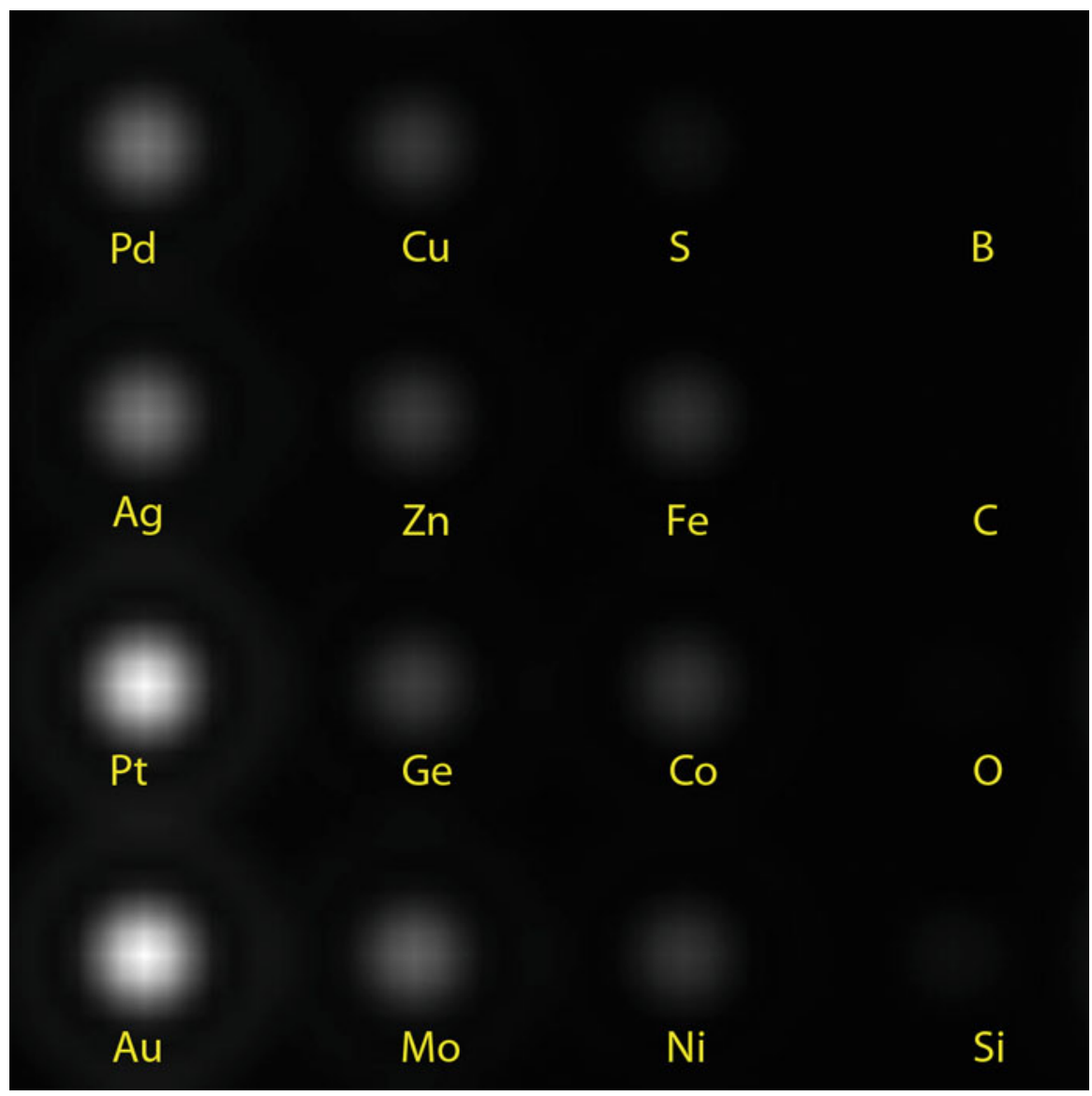

Fig. 1.8 STEM-simulated micrograph showing the intensity signal due to the presence of atoms of different elements

"The answer is that the brain supplies the missing information, information about the world we evolved in and how it reflects light. If the visual brain "assumes" that it is living in a certain kind of world — an evenly lit world made mostly of rigid part with smooth, uniformly colored surfaces - it can make good guesses about what is out there." [16]

The interpretation of the electron microscope images must be made under the same kind of assumptions: As a first approximation, one considers that the nanostructures are laying on an even surface (usually carbon), and that in inner regions of the nanostructure and far from twin boundaries, point-defects and dislocations, the atomic arrangement is close to that of the bulk. Furthermore, it is assumed that the electron beam and the nanoparticle, like the human visual brain, live in a certain kind of world where some geometries are more expected than others. 


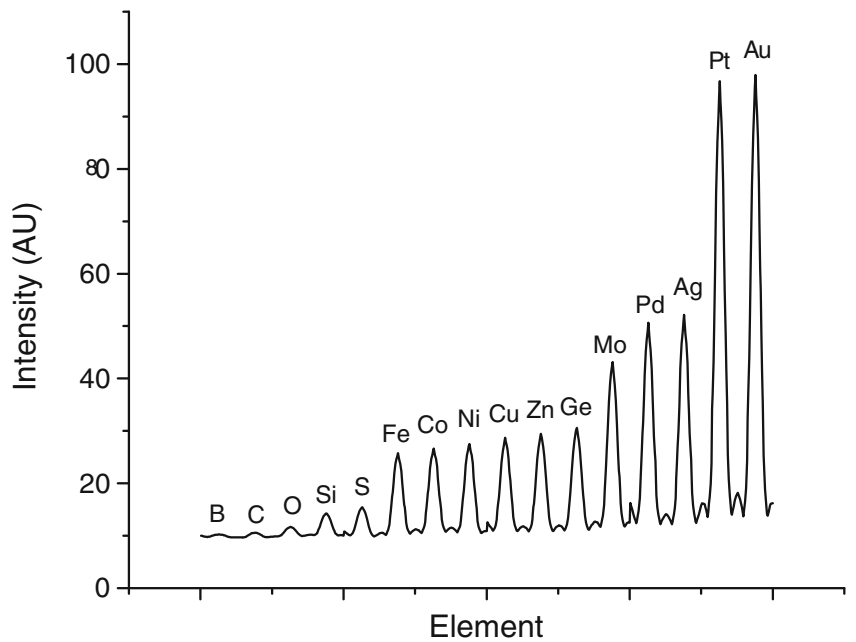

Fig. 1.9 HAADF-STEM intensity signal (arbitrary units) for several chemical elements

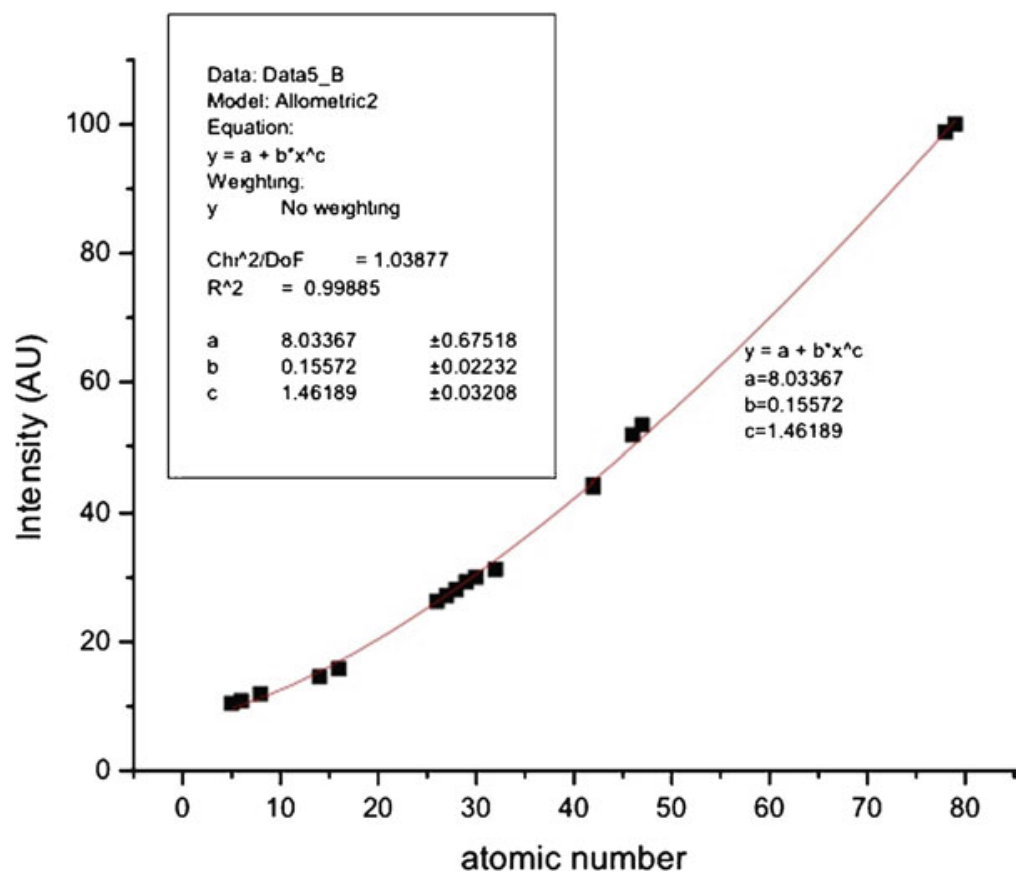

Fig. 1.10 STEM intensity vs. atomic number obtained by STEM simulation. The least-squared regression predicts an intensity dependence as $Z^{1.46}$ 


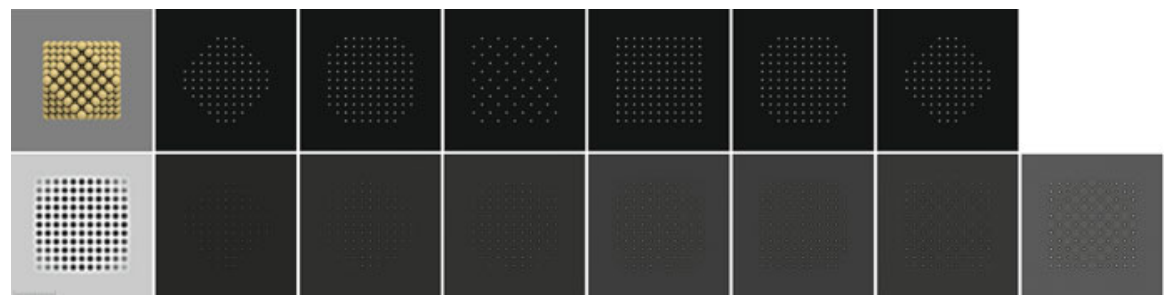

Fig. 1.11 Upper row: Atomistic model of a cuboctahedral gold nanoparticle, and the calculated projected potentials for each slice. Lower row: Output wavefunctions of each slice. The model was divided into ten slices, but only the nontrivial ones are presented

As it was mentioned before, the approximations used in the different simulation techniques are strictly true only when the sample is thin enough for the linear assumption to be correct. One of the most common approaches to implement HRTEM simulation take this assumption as the starting point, and for instruction purposes, the discussion of other difficulties will be avoided. The reader is encouraged to read the excellent text by Kirkland on this matter [10]. On the remaining of this section, we will concentrate on the use of the multislice method for the simulation of HAADF-STEM micrographs, and on the technical issues that may simplify-or make practically impossible — an interpretation of a simulated image.

\subsubsection{The Problem of Slicing a Sample}

As was pointed out before, in the multislice method the sample is dissected into several slices, each treated independently through an averaged projected potential. The selection of the number of slices is a tricky job, and it will depend on the thickness of the sample (the size of the nanostructure in the case of metal nanoparticles), the orientation, and the computational capabilities. The projected potential is calculated for each slice, and the corresponding output wavefunction is obtained. Figure 1.11 shows the result of the calculation of potentials and wavefunctions for a model of a gold cuboctahedral nanoparticle. The model was dissected into ten slices (only the relevant ones are represented in the figure).

If the number of slices is increased, the number of projected potentials will increase as well. This will reflect on the final result of the image, as can be seen in Fig. 1.12, where a model of an icosahedral gold particle-shown in Fig. 1.12awas used to generate two simulated STEM micrographs, being the only difference between both the number of slices, 10 in (b), 20 in (c). It is not a simple task to find out which of the images follows in a better way the experimental one, since the orientation and lattice parameters must be considered.

For the rest of the chapter, in order to compare the figures related with the Z-contrast in STEM, we will use STEM simulations where the samples have been 

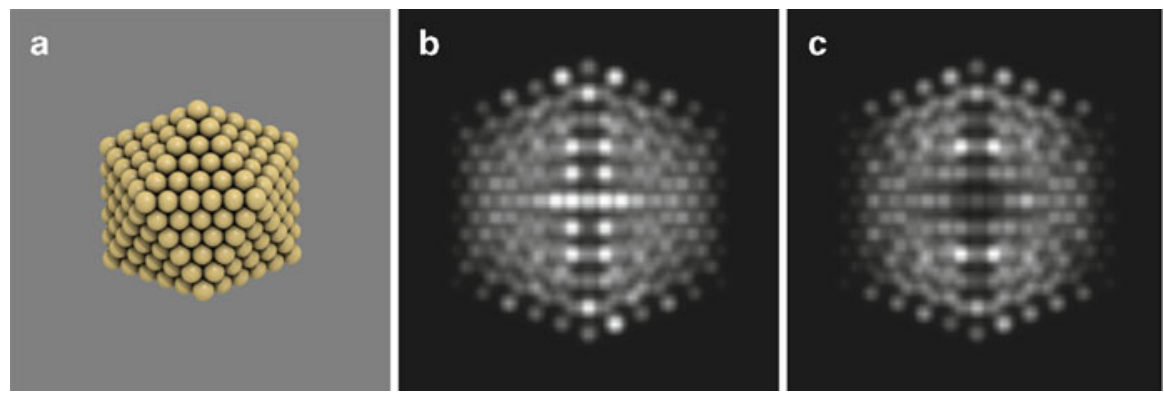

Fig. 1.12 (a) Atomistic model of an icosahedral gold nanoparticle; (b) STEM simulation of the nanoparticle, using ten slices; (c) STEM simulation of the nanoparticle, using 20 slices

divided into ten slices, irrespective of the model being used in the simulations. We encourage the reader interested in the multislice method to dedicate generous amounts of time to try out different slicing possibilities.

\subsubsection{Orientations}

One of the first pieces of information that appear when analyzing electron micrographs of metal nanostructures is that there is a remarked tendency for the particles to appear with some specific geometries. The reason for this tendency is mostly energetic (apparently these geometries imply the best compromise between surface energy, conservation of crystal structure, energy of twinning boundaries, and surface/volume ratio), but it is well established that the specific geometry depends not only on the chemical constitution of the particle, but also on its size, and on the specific details of the kinetics involved in the synthesis process [2]. Typical geometries in mono and multimetallic particles include decahedra, icosahedra, tetrahedral, and cuboctahedra. How evident the shape of a particle is depends on how close is the particle to the ideal geometry, and also on the orientation of the particle with respect to the electron beam. Figure 1.13 exemplifies this for the case of a small icosahedral gold particle.

\subsubsection{Chemical Composition}

Returning back to the issue of the Z-contrast dependency on STEM, we take advantage of the previous figure to compare it against the simulated STEM micrographs of particles of the same size and shape, but with different composition. Figure 1.14 shows the STEM-simulated images of an icosahedral $\mathrm{Au}_{\text {core }}-\mathrm{Pd}_{\text {shell }}$ particle, at the same orientations used in Fig. 1.13. The orientation of (b) and ( $\left.b^{\prime}\right)$ 


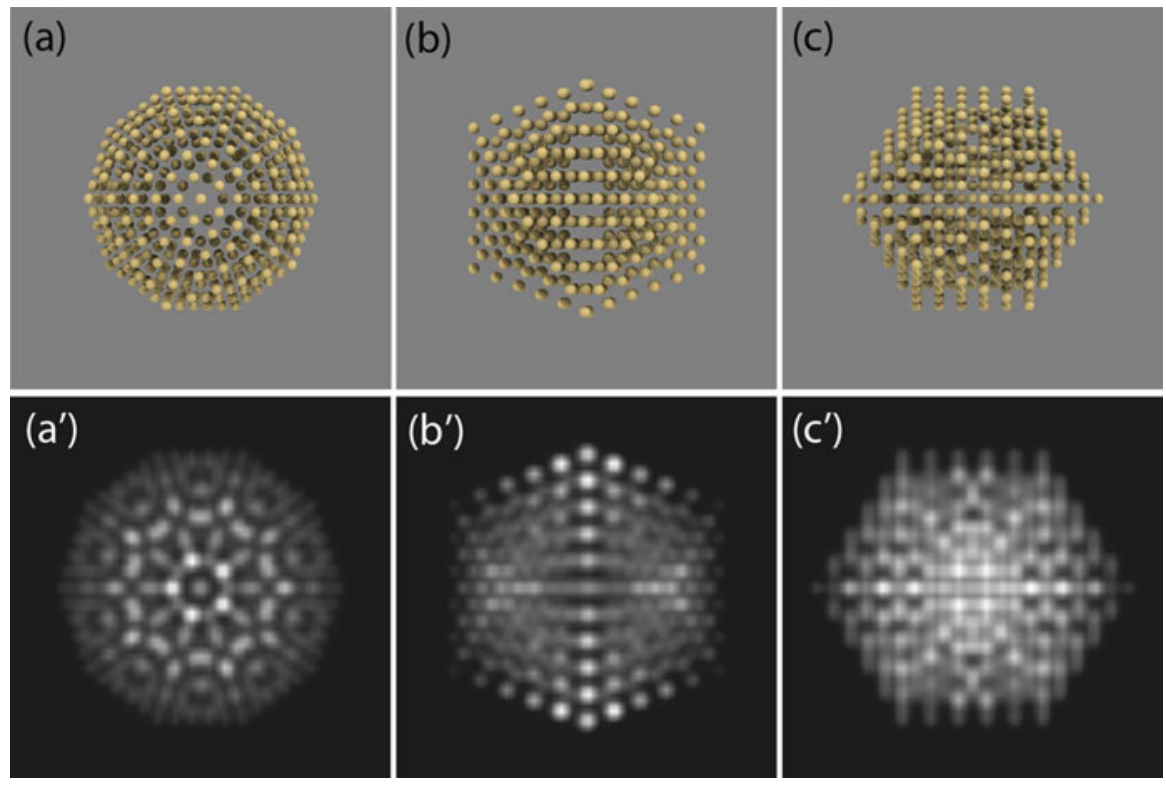

Fig. 1.13 Icosahedral gold nanoparticle at three different orientations, and their corresponding STEM-simulated images

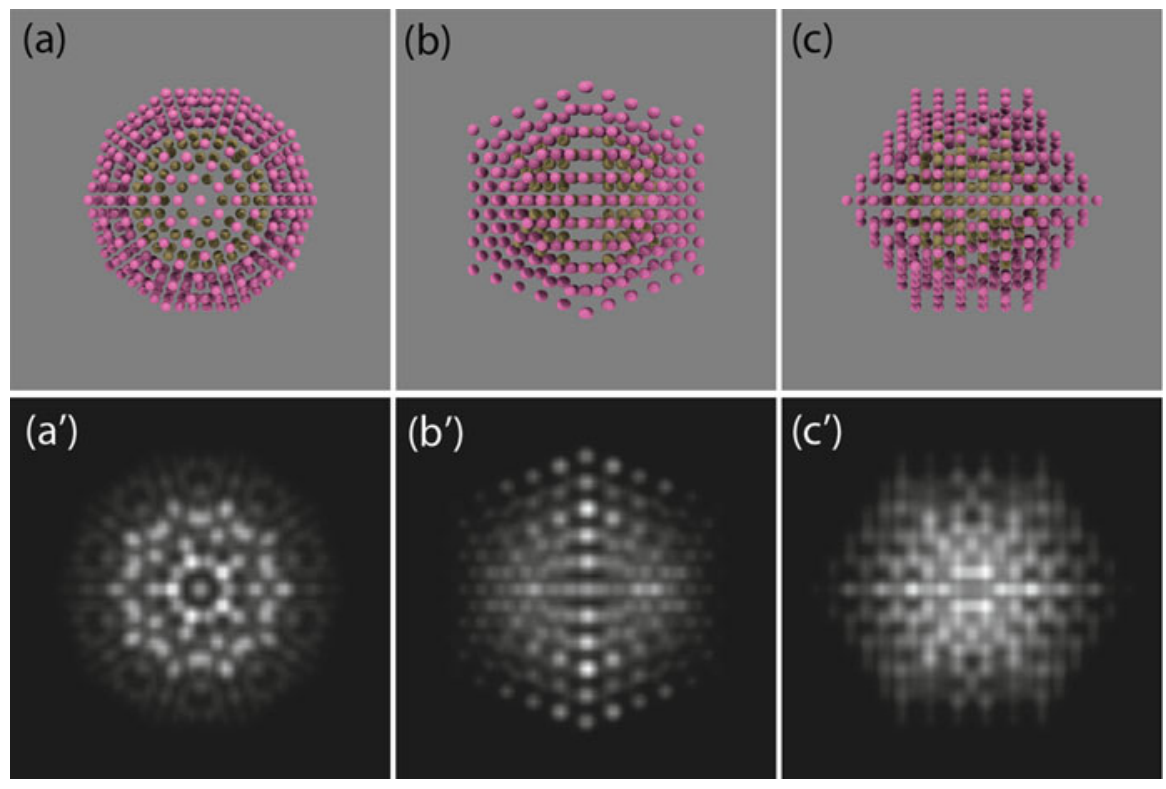

Fig. 1.14 $\mathrm{Au}_{\text {core }}-\mathrm{Pd}_{\text {shell }}$ nanoparticle at three different orientations, and their corresponding STEM-simulated images 


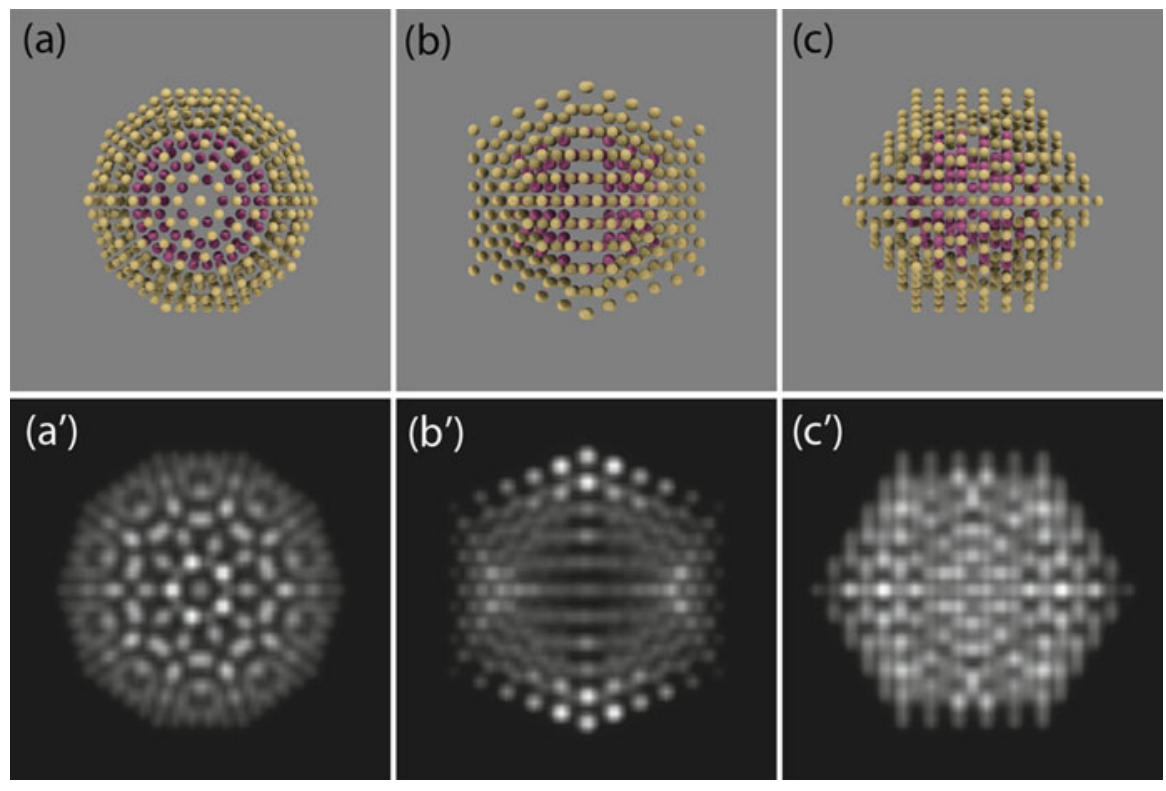

Fig. 1.15 $\mathrm{Pd}_{\text {core }}-\mathrm{Au}_{\text {shell }}$ nanoparticle at three different orientations, and their corresponding STEM-simulated images

is $30^{\circ}$ with respect to (a), and (c) and $\left(c^{\prime}\right)$ are rotated $90^{\circ}$ with respect to (a). The difference in intensity between core and shell are evident.

A particle with the opposite relative concentration is used in the STEM simulations shown in Fig. 1.15. Here, the particle's core and shell are not as easily distinguishable as in the previous case, since, unlike the opposite composition, the thickness of the gold regions compensates the difference in the atomic number of the elements (see Fig. 1.16).

The signal generated by a true Au-Pd nanoalloyed particle is different enough from the core-shell cases to be directly identified. Figure 1.17 shows the results of the STEM simulations for this particle. The relative composition of this particle is close to $50 \%-50 \%$, and both species are distributed randomly along the whole of the particle.

How can the Z-contrast capabilities of HAADF-STEM be used in the investigation of real metal particles? As an example, consider the Au-Co particle presented in Fig. 1.18a. This particle was produced by the coalescence of a gold particle with a cobalt particle, both originally synthesized by inert gas condensation and deposited on a carbon substrate. After deposition, the system was subjected to a heating process [17]. The particle in Fig. 1.18a is shown in false colors to remark the differences in intensity (red is more intense, blue is less intense). Chemical analysis showed that the low intensity regions were rich in cobalt and oxygen - the cobalt oxidized with the thermal treatment)—while the high intensity region was rich in gold. A model consisting on a decahedron with a gold core and a $\mathrm{CoO}$ external region was prepared 
Fig. 1.16 Diagram of a $\mathrm{Pd}_{\text {core }}-\mathrm{Au}_{\text {shell }}$ nanoparticle, showing that gold volume fraction explains the poor contrast between core and shell in a STEM micrograph
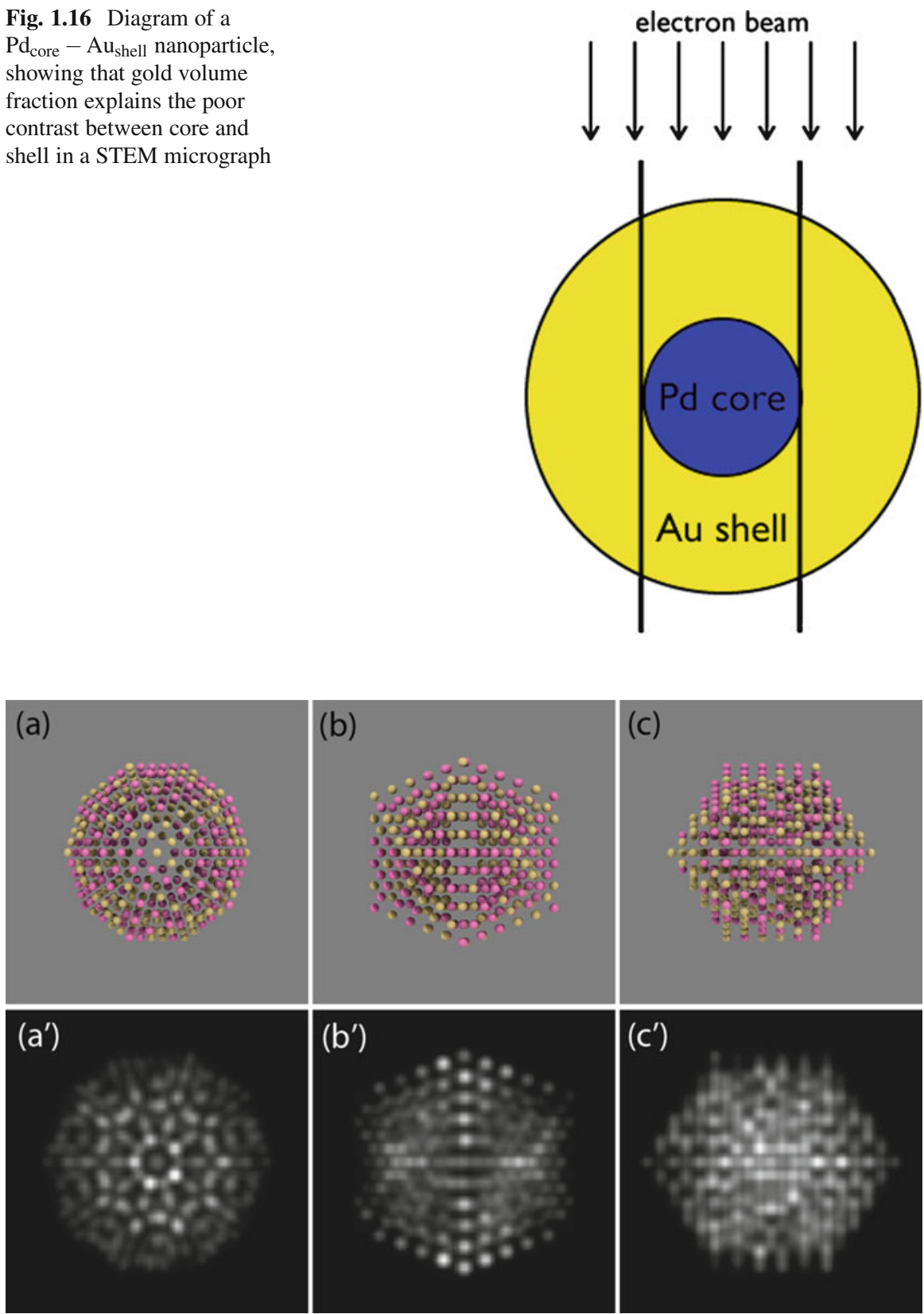

Fig. 1.17 Nanoalloyed Au-Pd icosahedral nanoparticle at three different orientations, and their corresponding STEM-simulated images 


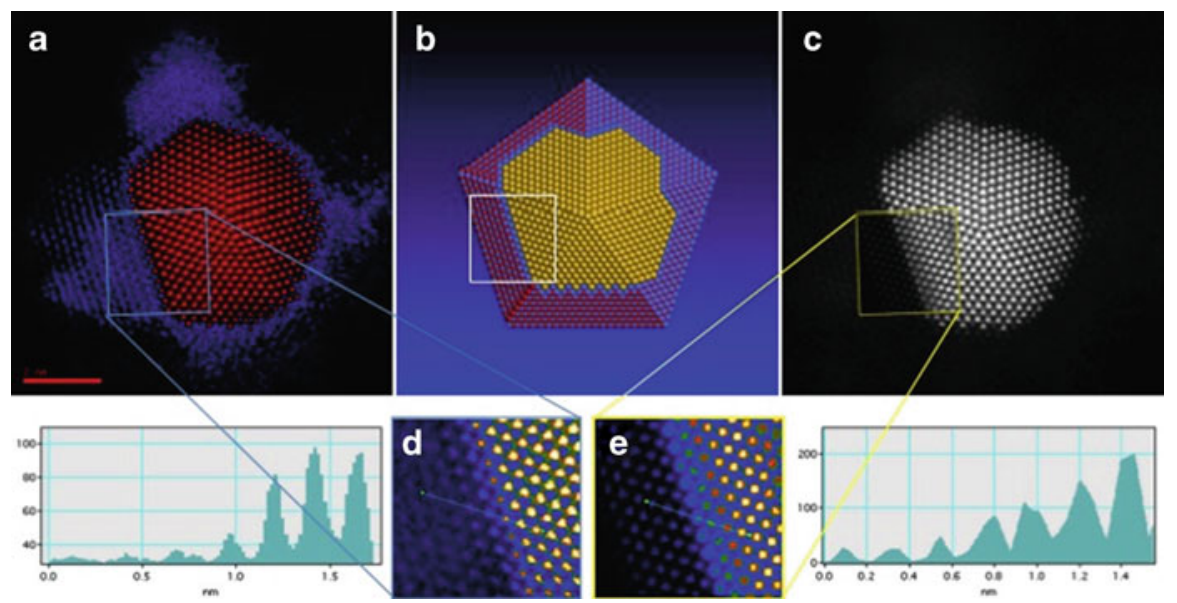

Fig. 1.18 (a) STEM micrograph of a Au-Co nanoparticle, shown in false colors (red: high intensity, blue: low intensity). (b) Atomistic model used to describe the particle on (a); yellow spheres represent gold, blue spheres are cobalt, red spheres are oxygen. (c) The small squared region marked in (a) is substituted for the STEM simulation of the squared region marked in (b), and (d) the real micrograph of this region is compared against (e) the simulated one. The real and simulated line intensity profiles are also shown for comparison. Adapted from [17]. Reproduced with permission from The Royal Society of Chemistry (RSC)

to investigate the Co-Au interface; this model is shown in Fig. 1.18b. The model does not intend to reproduce exactly the shape of the particle, but to give a qualitative description of the particle. Figure 1.18c is a composite of the real image and a small section generated by STEM simulation; the corresponding real section is shown in Fig. 1.18d, and compared against the simulated micrograph (Fig. 1.18e). The two intensity profiles represented by lines in (d) and (e) are also shown for comparison. As can be seen, the simulated STEM micrograph succeeds in reproducing the main features of the real image. This result exemplifies the use of STEM simulations for an approximate description of composition and shape of metal particles.

Quantitative STEM simulation at atomic level is quite more complicated. Fluctuations in intensity on the background of a STEM micrograph can be of the same order of magnitude than the intensity generated by a single metal atom, and in multimetallic systems the problem becomes combinatorial. Nevertheless, the use of single-tilt tomography holders altogether with $C_{s}$ correctors and 3D image reconstruction algorithms allow a very precise description of shapes and structures in nanoparticles, even at atomistic level [18-20].

\subsubsection{Thermal Effects}

The scattering strength is controlled by the effective form factor that depends on the value of the Debye-Waller factor, and variations in this factor may strongly affect 

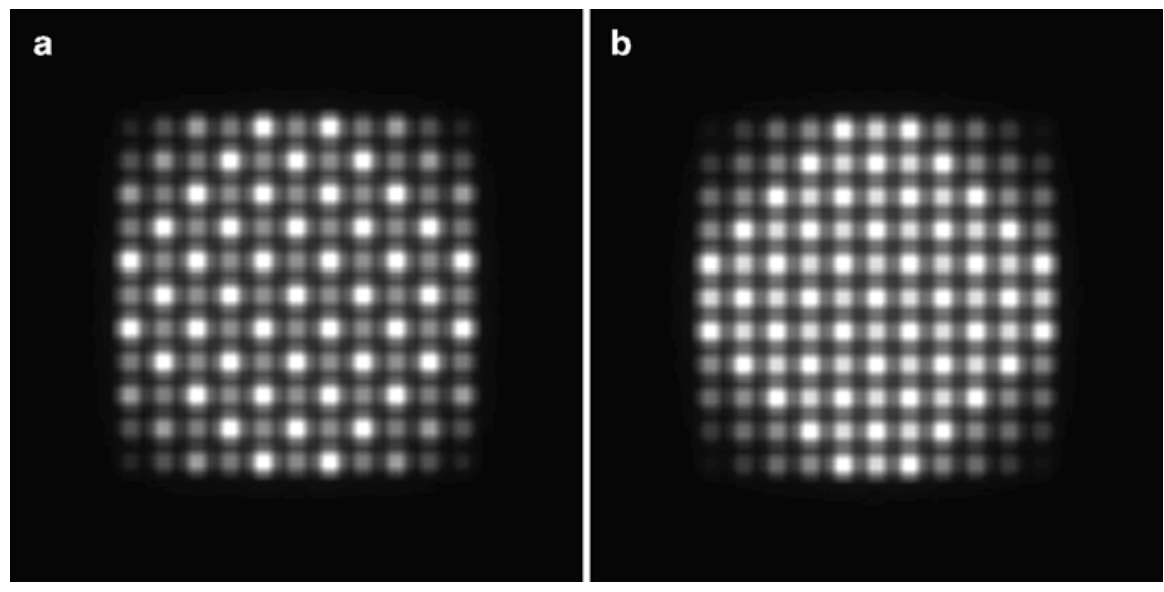

Fig. 1.19 Comparison between two simulated STEM micrographs of a cuboctahedral nanoparticle. (a) Debye-Waller set to zero. (b) Debye-Waller set at 0.69

the STEM signal. It is usual to find that an electron microscopy simulation program calculate the effective form factors through the Mott formula [21]. The DebyeWaller factor is not necessarily the same at the nanoscale than the bulk value. Buffat [22] measured a decrease in the Debye-Waller factor in gold particles ranging from 0.85 in $20 \mathrm{~nm}$ particles, to a value of 0.69 in $2 \mathrm{~nm}$ particles. We used this value to simulate a gold cuboctahedral particle and compare the result against a simulation where the Debye-Waller factor was set to zero. Both images are shown in Fig. 1.19. As can be noted, the thermal effects may be a determining factor on the contrast in ultra-high resolution STEM micrographs, and should not be underestimated.

\subsubsection{Interatomic Distances}

One of the most straightforward uses of TEM and STEM is the measurement of distances based on the intensity peaks (or valleys in bright field imaging) on the micrographs. For this direct kind of measurements, it is assumed that the spatial frequencies are uniformly transferred, this is, that the transfer function does not change its sign in the range of spatial frequencies that correspond to interatomic distances. Even if this condition is accomplished, this does not warranties the match between the measured and the real distances, since also the orientation of the sample has to be considered. The interpretation, though, is not necessarily straightforward: First, even in the best tuned-up TEMs, the background intensity is not homogeneous, and this can complicate the interpretation of distance measurements at the vicinity of the borders of a particle. Second, usually the interplanar distances are measured as an average over several planes, since the variance in 

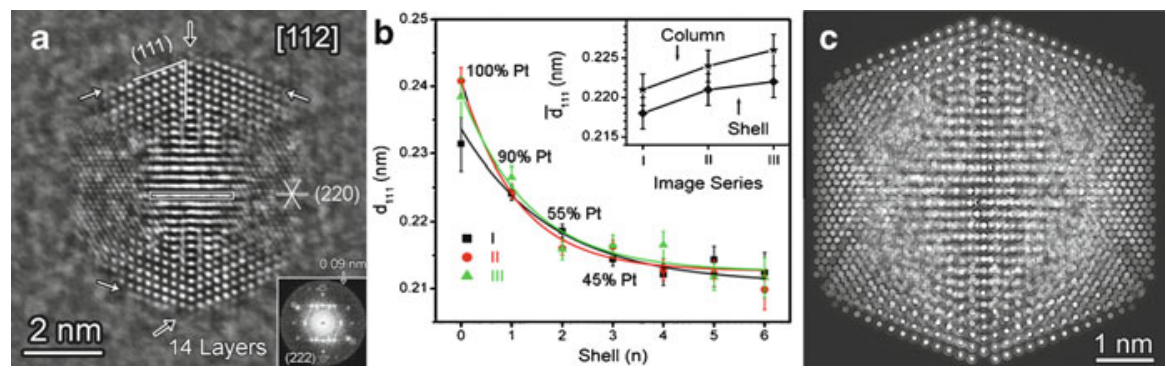

Fig. 1.20 (a) Sub-Angstrom resolution FePt icosahedral nanoparticle. (b) Interplanar (111) distances vs. position of shell (shell 0 is the external shell). (c) TEM simulation of model nanoparticle. In order to reproduce the measurements of the interplanar distances, the distribution of Pt must follow the percentages shown in (b). Reprinted with permission from [23]. Copyright 2009 American Chemical Society

the size of a spot that represents a single atomic column is on the same order of magnitude as the interplanar distance itself. High resolution STEM simulations can be used to verify the experimental measurements, if an appropriate atomistic model is taken for granted. Consider for example the case of FePt icosahedral nanoparticles [23], as the one shown in Fig. 1.20. FePt nanoparticles with sizes on the range of $[5,6] \mathrm{nm}$ were produced by an inert gas condensation, which implies that there is no passivation agent on the surface of the particles. Their structure was analyzed as icosahedral with shell periodicity. The sub-Angstrom resolution was achieved by focal series reconstruction, and it was enough to extract the position of individual atomic columns with a precision of about $0.002 \mathrm{~nm}$, and to measure shell spacing differences of $0.02-0.03 \mathrm{~nm}$. The measurements showed that the external shells of the nanoparticle were more spaced that the inner ones, a trend that could be interpreted as a consequence of the segregation of Pt to the external shells (see Fig. 1.20b). Comparative Molecular Dynamics calculations used jointly with electron microscopy simulations (Fig. 1.20c) confirmed that the structural relaxations could be attributed to Pt segregation at the outer shells generating a specific concentration gradient.

\subsection{STEM, Geometrical Phase Analysis, and Its Range of Validity}

Sub-Angstrom resolution in TEM and STEM, altogether with the ability of tilting the samples to orientations where a specific zone axis coincides with the direction of the electron beam, opens the possibility of analyzing the structures from a geometric perspective in order to obtain a strain map of the lattice. This possibility is particularly important in metal structures with Platonic and Archimedean polyhedral shapes, where strains may not be evenly distributed. An archetypical example of 
this is the decahedron, that in order to exist as a closed structure formed by five tetrahedra, it has to deform its atomic lattice, originating internal strain. A commonly used method to measure strains directly from high resolution micrographs is the one called geometric phase analysis, or GPA in short [24]. The method, which in principle can be applied to any image with two or more sets of periodicities, is based on the local measurement of amplitude and geometric phase in reciprocal space, i.e., by the measurement of the displacement of lattice fringes in the Fourier transform of the image. There exist free and paid versions of the GPA code, both implemented to work as add-ons of the DigitalMicrograph suite [25]. The measurement procedure, in a nutshell, is as follows: (1) The power spectrum of the image (the squared modulus of the Fourier transform of the micrograph) is calculated; each periodicity present in the image will generate a bright spot in the power spectrum, and the dispersion of the spot will be at least partially due to variations in the structure. (2) A region of interest enclosing a particular bright spot is selected; this will be the reciprocal area where the phase variations will be measured. (3) The phase image is calculated following the relation

$$
P_{g}(r)=-2 \pi g \cdot u(r),
$$

where $g$ is the reciprocal vector that defines the periodicity. (4) A reference area is chosen, and the phase image is recalculated, referenced to this area. The ideal reference area must be a region where the periodicity is not distorted, i.e., where the non-referenced phase image does not change abruptly. (5) The process is repeated for a second region of interest, since two sets of lattice fringes are needed for the calculation of strains. The displacement vector is expressed in terms of the base vectors defining the two periodicities:

$$
u(r)=-\frac{1}{2 \pi}\left[P_{g 1}(r) a_{1}+P_{g 2}(r) a_{2}\right],
$$

where

$$
g_{i} \cdot a_{j}=\delta_{i j} .
$$

(6) The strain tensor is calculated:

$$
\varepsilon_{i j}=\frac{1}{2}\left(\frac{\partial u_{i}}{\partial u_{j}}+\frac{\partial u_{j}}{\partial u_{i}}\right),
$$

and (7) rotations of the lattice are calculated:

$$
\omega_{i j}=\frac{1}{2}\left(\frac{\partial u_{j}}{\partial x_{i}}-\frac{\partial u_{i}}{\partial x_{j}}\right) .
$$

Johnson et al. [26] calculated in this way the strain distribution in a decahedral gold 17-nm-radius nanoparticle, using a high resolution Aberration-corrected TEM 


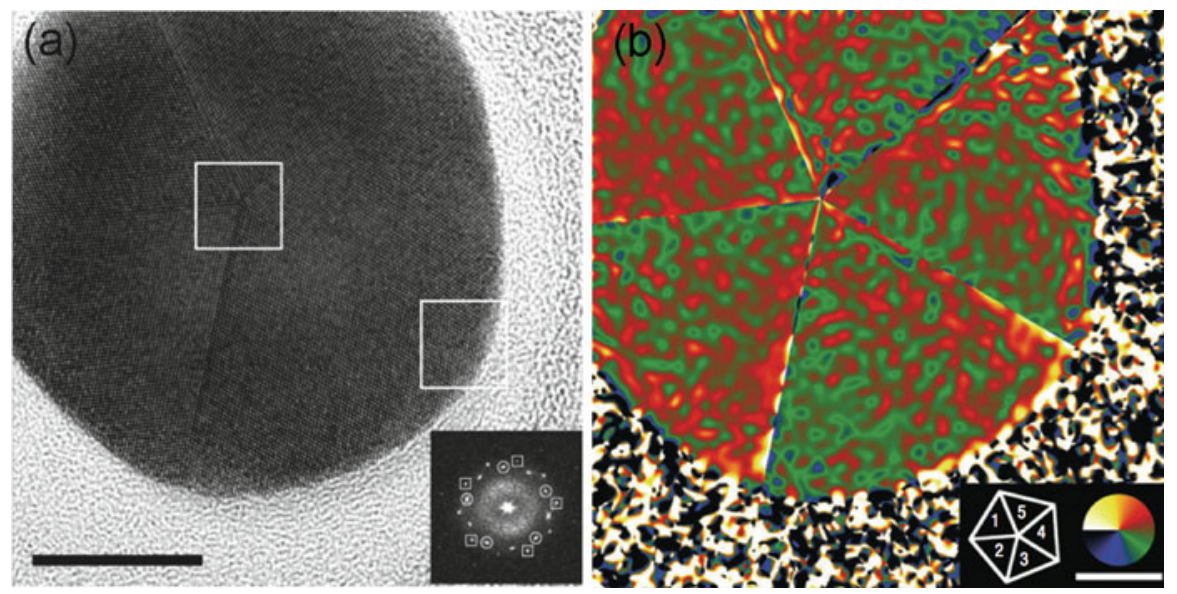

Fig. 1.21 (a) TEM micrograph of a fivefold gold nanoparticle. The scale bar is $10 \mathrm{~nm}$ long. (b) Shear strain map obtained by geometric phase analysis. Adapted by permission from Macmillan Publishers Ltd: [26], copyright 2007

image obtained with a Tecnai F20 ST (FEI) microscope. The results are shown in Fig. 1.21.

They found that, in order to close the 7.35 degree gap left by five perfect tetrahedra, each tetrahedron is strained nonhomogeneously (see Fig. 1.21b).

Is it possible to use STEM micrographs to generate strain maps by GPA? It would be interesting to give it a try, since the contrast in STEM may be as good as or even better than that of conventional TEM, and the interpretation of STEM is more direct than the interpretation of TEM, but the scanning process may introduce some artifacts in the geometric analysis. This source of error can be partially overcome by aligning the scan parallel to a principal axis of strain [27], or by rotating the STEM image and then repeating the GPA process.

The use of GPA on STEM micrographs is exemplified in Fig. 1.22. Here, a decahedral gold particle very well orientated along its fivefold axis was imaged by HAADF-STEM, and subjected to GPA analysis using the GPA Phase plugin for DigitalMicrograph by HREM Research, based on the original algorithm by Hytch [24]. Here we can note a qualitatively different strain distribution from the particle of Fig. 1.21; in this case, the strain is distributed nonhomogeneously in each tetrahedron, and some almost horizontal stripes of higher strain than the rest of the particle appear crossing the frontiers between adjacent tetrahedra. Whether these stripes are related to the scanning process is still uncertain, and here we present the results to remark the need of double checking the GPA results by improving the statistics of the measurement, repeating the GPA process to several micrographs of the same particle, rotating the micrographs (or even the sample), and carefully filtering the images. 

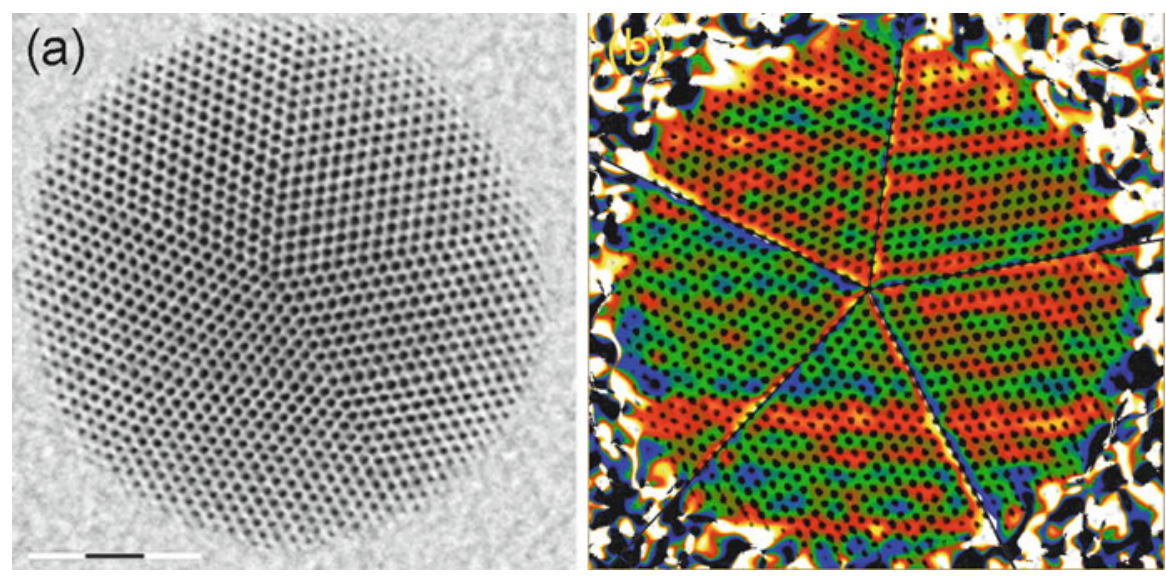

Fig. 1.22 (a) HAADF-STEM micrograph of a fivefold gold nanoparticle. (b) Shear strain map obtained by geometric phase analysis

\subsection{Conclusions}

Electron microscopy at sub-Angstrom resolution has reached such a high level of precision that we can investigate the composition and structure of metal nanoparticles in an atom-by-atom fashion. This gives an invaluable capacity for the study of the interaction of metal and alloy nanostructures with biological tissue, since now it is possible to look for structure/function correlations in real time, and with a resolution high enough to investigate individual bonds. The simultaneous use of real and simulated microscopy makes the process of interpreting a micrograph more science and less craftsmanship, and gives the conclusions extracted from the measurements a more quantitative and less speculative character. Some conditions must be fulfilled to take full advantage of the top electron microscopy facilities when studying metal nanostructures: The capacity of preparing high quality samples, a fair understanding of the interaction between the sample and the electron beam, a conscious awareness of the capabilities and limitations of the different instruments and techniques, and the familiarity with different analysis approaches. And, above all, the essential element: an experienced and skillful microscopist.

Acknowledgments The authors would like to acknowledge The Welch Foundation Agency Project \# AX-1615, "Controlling the Shape and Particles Using Wet Chemistry Methods and Its Application to Synthesis of Hollow Bimetallic Nanostructures." The authors would also like to acknowledge the NSF PREM Grant \# DMR 0934218, Title: Oxide and Metal Nanoparticles-The Interface between life sciences and physical sciences. The authors would also like to acknowledge RCMI Center for Interdisciplinary Health Research CIHR. The project described was supported by Award Number 2G12RR013646-11 from the National Center for Research Resources. The content is solely the responsibility of the authors and does not necessarily represent the official views of the National Center for Research Resources of the National Institutes of Health. 


\section{References}

1. Ercolessi F, Andreoni W, Tosatti E (1991) Melting of small gold particles: mechanism and size effects. Phys Rev Lett 66:911-914

2. Ferrando R, Jellinek J, Johnston RL (2008) Nanoalloys: from theory to applications of alloy clusters and nanoparticles. Chem Rev 108:845-910

3. Barnard AS, Young NP, Kirkland AI, van Huis MA, Xu H (2009) Nanogold: a quantitative phase map. ACS Nano 3:1431-1436

4. Antúnez-García J, Mejía-Rosales S, Perez-Tijerina E, Montejano-Carrizales JM, JoséYacamán M (2011) Coalescence and collisions of gold nanoparticles. Materials 4:368-379

5. Carter CB, Williams DB (2009) Transmission electron microscopy: a textbook for materials science. Springer, New York

6. Fernández-Navarro C. et al. (2007) On the structure of Au/Pd bimetallic nanoparticles. J Phys Chem C 111:1256-1260

7. Yuan D, Gong X, Wu R (2008) Peculiar distribution of Pd on Au nanoclusters: First-principles studies. Phys Rev B 78: 035441

8. Gómez-Rodríguez A, Beltrán-del-Río LM, Herrera-Becerra,R (2009) SimulaTEM multislice simulations for general objects. Ultramicroscopy 110:95-104

9. Pennycook S (1989) Z-contrast STEM for materials science. Ultramicroscopy 30:58-69

10. Kirkland EJ (1998) Advanced computing in electron microscopy, vol. 250. Springer, New York

11. CtfExporer. CtfExporer (Sidorov, M. V.:).at http://www.maxsidorov.com

12. Stadelmann P. JEMS. http://cimewww.epfl.ch/people/stadelmann/jemsWebSite/jems.html

13. Ishizuka K (2001) A practical approach for STEM image simulation based on the FFT multislice method. Ultramicroscopy 90:71-83

14. Ishizuka K (1998) Multislice implementation for inclined illumination and convergent-beam electron diffraction. Proc Intl Symp Hybrid Anals

15. Wall J, Langmore J, Isaacson M, Crewe AV (1974) Scanning transmission electron microscopy at high resolution. Proc Natl Acad Sci USA v71: 1-5

16. Pinker S (1997) How the mind works. W. Norton \& Company, New York

17. Mayoral A, Mejía-Rosales S, Mariscal MM, Perez-Tijerina E, José-Yacamán M (2010) The Co-Au interface in bimetallic nanoparticles: a high resolution STEM study. Nanoscale 2:2647

18. Gontard LC, Dunin-Borkowski RE, Gass MH, Bleloch AL, Ozkaya D (2009) Threedimensional shapes and structures of lamellar-twinned fcc nanoparticles using ADF STEM. J Electron Microsc 58:167-174

19. Kimoto K et al. (2007) Element-selective imaging of atomic columns in a crystal using STEM and EELS. Nature 450:702-704

20. Muller DA (2009) Structure and bonding at the atomic scale by scanning transmission electron microscopy. Nat Mater 8:263-270

21. Ishizuka K (2002) A practical approach for STEM image simulation based on the FFT multislice method. Ultramicroscopy 90:71-83

22. Buffat P, Borel JP (1976) Size effect on the melting temperature of gold particles. Phys Rev A 13:2287

23. Wang R et al. (2009) FePt Icosahedra with magnetic cores and catalytic shells. J Phys Chem C 113:4395-4400

24. Hytch M, Snoeck E (1998) Quantitative measurement of displacement and strain fields from HREM micrographs. Ultramicroscopy 74:131-146

25. Mitchell DRG, Schaffer B Scripting-customised microscopy tools for Digital Micrograph (TM). Ultramicroscopy 103:319-332

26. Johnson CL et al. (2007) Effects of elastic anisotropy on strain distributions in decahedral gold nanoparticles. Nat Mater 7:120-124

27. Diercks D, Lian G, Chung J, Kaufman M (2011) Comparison of convergent beam electron diffraction and geometric phase analysis for strain measurement in a strained silicon device. J Micros 241:195-199 